\title{
¿SE ESTÁ TORNANDO AMARGO \\ EL MILAGRO MEXICANO?
}

\author{
GustaV RaNis \\ Yale Growth Center
}

MÉxICo APARECE invariablemente en la lista del grupo selecto de narraciones de casos de desarrollo de la posguerra que han logrado el éxito. Como prueba de ello, con gran frecuencia se cita una tasa de crecimiento elevada y sostenida (mayor que 6\%) a lo largo de un período prolongado (desde fines de los años treinta), acompañada de estabilidad política y acceso rutinario, casi automático, al mercado internacional de capitales (en especial al de los Estados Unidos). Pocos países en desarrollo, y ninguno de América Latina, han podido igualar esta evolución extraordinaria.

Para quien quiera escuchar, existen desde luego otras opiniones. Éstas son la voz crítica de una minoría aún, que recuerda el ethos revolucionario del pasado y señala que tal progreso ha sido logrado al precio de disparidades todavía mayores en la distribución del ingreso y niveles cada vez más intolerables de desempleo y subempleo. En gran parte de esta crítica está implícito el supuesto de que, mientras la estrategia seguida hasta ahora ha estado bien engranada al logro de un crecimiento máximo, las presiones políticas y sociales han de forzar ahora un cambio en los objetivos sociales subyacentes.

En este documento se intenta poner en duda las dos proposiciones siguientes: primero, que la experiencia del desarrollo mexicano se ha aproximado, en el pasado, a una trayectoria óptima desde el punto de vista del objetivo tradicional del crecimiento $\mathrm{y}$, segundo, que lo que hace falta ahora es una trayectoria que responda a las demandas de un nuevo conjunto de objetivos sociales. Expresado en forma más positiva, este análisis del desarrollo mexicano conduce a la conclusión de que, en términos de los objetivos de crecimiento tradicionales, existen serias deficiencias subyacentes, que han estado en operación durante un buen tiempo, aunque tal vez se hayan agudizado recientemente. De acuerdo con este punto de vista, lo que se requiere hoy en día para enfrentarse al problema surgido no es, cuando menos en primera instancia, una reorientación de los objetivos que consista en "bajar del trono al PBI" sino un cambio fundamental de estructuras que coloque al PBI en un trono más sólido. Un cambio en la forma en que el PBI ha sido generado habría redituado un mayor empleo y una distribución mejor del ingreso sin sacrificar el producto - y ello puede hacerse hoy. Esto no quiere decir que el clamor actual por mayor igualdad y un crecimiento con una base más amplia deba o de hecho pueda descartarse, sino más bien que debe examinarse el supuesto usual de que los objetivos de crecimiento y empleo son necesariamente competitivos. En nuestra opi. 
nión, la situación es la opuesta, durante varios decenios. México ha estado operando fuera del óptimo dentro de su frontera de posibilidades.

En este trabajo se pretende analizar el caso mexicano dentro de tal perspectiva histórica y dentro de un marco agregativo, tal vez informal, de teoría de crecimiento. Primero se presenta brevemente dicho marco y se investiga la experiencia histórica mexicana en ese contexto y posteriormente se da atención a las circunstancias presentes y a las conclusiones de política para el futuro que emergen de nuestro análisis.

La mayoría de los países en desarrollo, entre ellos México, heredaron un pasado colonial caracterizado por la existencia de un sector de enclave que proporcionaba materias primas, minerales, etc. al resto del mundo, mientras que recibía a cambio bienes de inversión que permitirían la expansión ulterior de tal enclave, por una parte, y por otra, bienes de consumo para satisfacer las necesidades de la élite gobernante, tanto nacional como extranjera. En estas condiciones el "resto de la economía", preponderantemente agrícola, en la medida en que no llenaba las necesidades del enclave en expansión, es decir, de proporcionarle trabajo y servicios, quedó muy a la zaga de la corriente principal del proceso de desarrollo. Esta era la situación durante el período porfirista previo a la revolución mexicana de 1910. Aunque el país había logrado su independencia política de España mucho antes, permaneció económicamente atado a los intereses de los Estados Unidos de Norteamérica y sirvió principalmente como abastecedor de minerales y otros productos primarios tales como tabaco, café, ganado y henequén, hasta los años treinta.

A este respecto muy general, México siguió más o menos el patrón de desarrollo latinoamericano colonial. El paso típico siguiente, dirigido a alcanzar la independencia económica y la construcción de un patrón de desarrollo nacionalista, ha consistido en la intervención de los gobiernos que se han insertado en èl patrón colonial de flujos de recursos y que ha recanalizado las ganancias tradicionales de divisas hacia el sector industrial de sustitución de importaciones. Este proceso bien conocido de sustitución de importaciones vía el uso de controles de divisas, de importaciones, deformación de los términos del intercambio en favor del sector industrial, etc., ha florecido plenamente en la mayoría del mundo subdesarrollado durante los años cincuenta y sesenta. En la mayor parte de América Latina el proceso comenzó antes, durante los años treinta, ya que parte del mundo en desarrollo que había logrado su independencia política antes, se vio luego sujeto a las ondas de choque internacionales de la gran depresión. Sin embargo, en el caso de México existe también una diferencia importante que debe hacerse notar y que es, en parte, responsable de su desenvolvimiento relativamente superior y responsable también de parte de la excitación (posiblemente prematura) citada al principio. Es decir que, mientras la mayoría del resto de los países de la región respondieron a la crisis de la gran depresión de los años treinta con una política de industrialización sustitutiva de importaciones -en ocasiones muy por encima de la capacidad y la dotación de calificación-, ${ }^{1}$ México se desvió de ese patrón y dio atención importante a la agricultura.

Específicamente, la administración de Cárdenas en los años treinta

1 Véase toda la literatura a la Prebisch, sobre sustitución de importaciones. 
no sólo introdujo la reforma agraria sino que construyó también buena parte de la infraestructura agrícola y se rehusó a permitir un deterioro en los términos del intercambio. La reforma agraria y las inversiones en irrigación, proporcionaron así las condiciones para un crecimiento relativamente sostenido de la productividad agrícola en México, en contraste con la mayor parte del resto de América Latina. Esta habilidad para permitir que el sector agrícola hiciera una contribución, en términos de un cambio de una base minera a una de producción de alimentos no tradicional, a la generación de recursos internos adicionales para el desarrollo, sirvió para prevenir un florecimiento temprano del delirio por la sustitución de importaciones, que emergió en todas partes y permitió el surgimiento de un patrón de crecimiento relativamente más equilibrado. En este sentido, tal vez más que en cualquier otro, haber tenido una revolución significó una diferencia real para el panorama económico mexicano.

El advenimiento de la sustitución de importaciones significa normalmente la reubicación de energías y recursos respecto de una orientación tradicional hacia las exportaciones y los servicios auxiliares a éstas, para la construcción de una industria nacional y sus servicios conexos. En el mejor de los casos, tal política da a los empresarios industriales la oportunidad de madurar, mientras se crea la infraestructura agrícola que eventualmente - es decir, en una fase posterior - permitiría participar al sector agrícola no orientado a las exportaciones. La sustitución de importaciones se demoró - no comenzó con todo su empuje hasta después de la segunda guerra - mientras se construía la infraestructura para acomodar el crecimiento de las exportaciones agrícolas comerciales, en especial en el norte del país.

Una segunda cuestión crítica de la estrategia de crecimiento, se enfoca por lo general hacia lo que sucede en la etapa primaria de sustitución de importaciones, cuando la tarea relativamente "fácil" de reemplazar las importaciones de bienes de consumo ha sido concluida, y la economía debe decidirse por una de dos trayectorias alternativas: continuar con la sustitución de importaciones, ahora en el área de los bienes intermedios y de capital, ambas áreas más intensivas de tecnología y de capital (esto es, de pasar a la etapa secundaria de importaciones); o desplazarse hacia una fase de crecimiento orientada a las exportaciones competitivas, una vez que el espíritu empresarial ha tenido la oportunidad suficiente de madurar. En el primer caso, el sector agrícola orientado a las exportaciones debe continuar proporcionando los medios para el programa de industrialización, ahora más costoso e intensivo de capital. En el segundo caso, el sector exportador industrial, creciente e intensivo en mano de obra, es llamado cada vez más a participar, en forma paralela a un crecimiento más equilibrado, vía una mayor participación del sector agrícola interno.

Debe quedar claro que en una cierta etapa del desarrollo del país puede ser necesaria una subfase de sustitución de importaciones. Probablemente se justificará por un tiempo que las utilidades estén por encima de lo normal, para compensar a los empresarios por sus riesgos, también por encima de lo normal. La herramienta utilizada con mayor frecuencia para llevar los recusos en las diecciones socialmente deseables, es la deformación de los precios relativos de los factores y de los productos. La cuestión se centra más bien sobre el tipo y la duración de la indus- 
trialización sustitutiva y la capacidad política para atenuar la temperatura y desplazarse a la siguiente subfase, justificable en términos de la dotación cambiante de los recursos de la economía.

Las reformas cardenistas de los años treinta apuntaban a una motivación temprana de la zona de influencia de la agricultura, donde la sustitución de importacionés habría de empezar más tarde, es decir, hasta la segunda guerra. La cubierta protectora natural de la guerra fue reemplazada luego por la protección tarifaria de la posguerra, concentrada en los textiles, calzado, ropa, alimentos, bebidas y tabaco. De hecho, según indica el cuadro 1, la sustitución primaria de importaciones, medida ya sea por la composición de las importaciones totales a por la proporción de los bienes de consumo industrial aún importados, quedó virtualmente terminada en los años cincuenta. En este momento se tuvieron todos los ingredientes necesarios para la transición hacia una trayectoria de desarrollo equilibrado, de economía abierta, donde la agricultura respaldaba a un sector industrial orientado hacia el exterior e intensivo de trabajo, al generar ahorros (tales como excedentes de alimentos) y liberar trabajo a salarios relativamente constantes. Así, de manera muy semejante al caso del Japón histórico y al de Taiwán contemporáneo, la atención relativamente mayor dedicada tempranamente a la agricultura, debió haber sido de gran ayuda para dar base a un crecimiento sostenido y equilibrado y orientado hacia un patrón de crecimiento y de exportación intensivo de mano de obra no calificada, y finalmente a uno intensivo de capital y de mano de obra calificada.

\section{Cuadro 1}

MÉXICO: SUSTITUCión PRIMARIA de IMPORTACIONES (Millones de pesos de 1960)

\begin{tabular}{|c|c|c|c|c|c|}
\hline & 1930 & 1940 & 1950 & 1960 & 1970 \\
\hline $\begin{array}{l}\text { Impor taotones de bienes de consumo } \\
\text { manufao turados, } v_{i}\end{array}$ & 601 & 354 & 1256 & 1626 & 1913 \\
\hline Importaciones totales, M & 2632 & 4024 & 9229 & 14831 & 21723 \\
\hline$x_{i} / M$ & 22.83 & 8.80 & 13.61 & 10.96 & 8.81 \\
\hline $\begin{array}{l}\text { Producoion interna de bienes do } \\
\text { oonsumo manufeoturados, } c_{i}\end{array}$ & 21715 & 26547 & 52239 & 100890 & 177155 \\
\hline oferta total, $c_{i}+M_{i}$ & 22316 & 26901 & 53495 & 102516 & 179068 \\
\hline$M_{i} / M_{i}+C_{i}$ & 2.69 & 1.33 & 2.40 & 1.61 & 0.11 \\
\hline
\end{tabular}

Fuente: Comisión Económica para América Latina, Anuarios de Comercio Exterior de México.

Sin embargo, inclusive durante la fase temprana de crecimiento equilibrado de los años treinta y cuarenta, existían dos tipos de problemas evidentes, los cuales, como veremos, sirvieron de bases a las dificultades más serias que aquejan ahora a la economía mexicana. Por una parte, inclusive entonces México tendió a moverse con cierta rapidez hacia las industrias de sustitución de importaciones intensivas de capital y de tecnología, por ejemplo papel, productos químicos y hierro y acero. Por la otra, las reformas y la construcción de la infraestructura agrícola se orientaron cada vez más a la agricultura intensiva, comercializada y de 
exportación, en la zona norte, y menos a la agricultura de subsistencia orientada al mercado interno, que abarca la mayor parte del país. Mientras que el carácter de la sustitución de importaciones se desplazó cada vez más hacia industrias que estimulan empresas conexas, el financiamiento del proceso pudo apoyarse cada vez menos en las exportaciones tradicionales (mineras en su mayor parte) y cada vez más en las exportaciones primarias no tradicionales tales como algodón, café y maíz, suplementadas por una contribución creciente del turismo. En otras palabras, sin duda debido, en parte, al entusiasmo fomentado por el éxito del período de sustitución "natural" de importaciones de la segunda guerra, y a la necesidad de sustituir las barreras artificiales de la posguerra, los orientadores de la política se movieron resueltamente hacia la sustitución de importaciones secundarias durante los años cuarenta y cincuenta, al mismo tiempo que se construía la infraestructura pesada requerida. Esto significó una ubicación de las divisas y los recursos internos en favor de las actividades del sector industrial, que demandaban sustancialmente mayor tecnología y capital. Para ilustrar esto, entre 1945 y 1955 la producción de bienes de capital aumentó $150 \%$, aunque a una escala reducida, mientras que los bienes de consumo aumentaron solamente en $57 \%$. Más aún, mientras la agricultura como sector continuó recogiendo los frutos de inversiones anteriores, al crecer la productividad agrícola en alrededor de $100 \%$ de 1945 a 1955 , estos totales se tornaron cada vez más decepcionantes, y en ellos la mayoría de los aumentos ocurrieron en el sector orientado en gran escala al mercado exterior y comercial. La producción agrícola no comercializada orientada al mercado interno aumentó su productividad en menos de $70 \%$ durante el mismo período.

Sin embargo, conviene hacer notar que las políticas mexicanas de industrialización desde la seguñda guerra hasta principios de los años cincuenta, pueden caracterizarse como de sustitución de importaciones por intervención indirecta, al contrario de una con uso intensivo de intervención directa. Esto significó que, en contraste con muchos países latinoamericanos en los años treinta y cuarenta y con la mayoría del resto del mundo subdesarrollado del período de la posguerra, México eludió el uso de controles de divisas y de crédito durante esa época y en su lugar acudió a controles tarifarios, subsidios y devaluaciones para permitir tanto una participación más amplia del sector privado como la retención de los vínculos relacionados a los precios con el resto del mundo.

Como consecuencia, el desenvolvimiento de México hasta principios de los años cincuenta fue muy bueno, en términos tanto de las normas de país de escaso desarrollo como de los países latinoamericanos. La sustitución de importaciones por intervención indirecta continuó dando una tasa de crecimiento sostenida y excepcionalmente elevada, unida a tasas de absorción de mano de obra satisfactorias en el sector no agrícola. La agricultura continuó beneficiándose de las inversiones previas, y mediante devaluaciones sucesivas, en 1948 y 1954, se mantuvo a raya un descenso potencialmente destructivo de la eficiencia industrial global, lo cual mantuvo una competitividad razonable con respecto al resto del mundo. De hecho, a estas alturas México tenía todavía la opción de cambiar a un patrón de desarrollo industrial orientado a las exportaciones. En lugar de ello, el país decidió, en algún momento a mediados de los años cincuenta, moverse a todo vapor hacia la etapa secundaria de sustitución de importaciones acudiendo cada vez más a los controles directos. Por 
ejemplo, después de 1954 se tomó la decisión de buscar más ayuda en la tasa de cambio para mantener la competividad internacional de la economía y por consiguiente de colocar una carga cada vez mayor sobre el control de las importaciones, del crédito y otros controles directos. Más del $80 \%$ de las importaciones manufactureras fueron controladas mediante licencias a mediados de los años cincuenta, que fue un decenio durante el cual se plantearon muchas de las semillas subyacentes a los problemas actuales -en mi opinión muy serios- de la economía mexicana.

Es clara la evidencia de que una vez que se tomó tal decisión, la tasa de crecimiento industrial descendió ligeramente, en relación con su nivel de los años cuarenta, y los requerimientos de capital para mantener ese crecimiento aumentaron en forma aguda. Mientras que en los años cuarenta la tasa de inversión fue menor a $10 \%$, y en los años cincuenta cercana a $15 \%$, para mantener una tasa de crecimiento igual o inclusive menor, la industria se tornaba cada vez más intensiva de capital, tanto en términos de la estructura del producto como de la tecnología. Tal como indica la gráfica 1, la relación capital/trabajo aumentó en forma sostenida entre 1940 y 1950 con una tasa de crecicimiento considerablemente mayor durante los años cincuenta y sesenta. Para prolongar la sustitución de importaciones y suministrarle combustible, hubo necesidad de construir una infraestructura pública nueva, en especial de irrigación y construcción de carreteras. A lo largo del tiempo, se destinaron más y más fondos a la agricultura norteña de gran escala orientada a las exportaciones, y cada vez menos hacia los sectores agrícolas privados de pequeña propiedad y ejidales, orientados al mercado interno. Un indicio de esto es que el cociente capital/trabajo en la agricultura en gran escala aumentó siete veces entre 1940 y 1960 , sólo 3.5 veces en los predios ejidales, y solamente 1.8 en la pequeña propiedad privada. ${ }^{2}$ Se disponía de irrigación, por mucho margen, solamente para la agricultura comercializada $y$, como consecuencia, la revo¿ución verde no llegó a vastas regiones del país. Otro sesgo importante en favor de la agricultura en gran escala ha sido hecho palpable por la agencia de precios de garantía (CONASUPo) que ha dispuesto sus centros de compra en forma desigual. A pesar de esto, la productividad total de los factores permaneció más alta en los predios más pequeños, ${ }^{3}$ pero la tasa de crecimiento global de la producción agrícola descendió de $5.8 \%$ en los años cuarenta a $4 \%$ en los cincuenta y los sesenta. Los aumentos de la productividad de la mano de obra agrícola descendieron de una tasa anual de $6 \%$ entre 1930 y 1940 , a $3.5 \%$ y menos en los años recientes, a pesar del aumento global en la intensidad del uso de capital. La productividad de la mano de obra industrial, que creció a una tasa anual de $5.6 \%$ en los años cuarenta, se elevó anualmente tan sólo $1.4 \%$ durante los años sesenta. El crecimiento de la productividad global de los factores, a nivel por encima de $2 \%$ en los años treinta, descendió a menos de $1 \%$ después de la segunda guerra, y a montos insignificantes en los cincuenta.

La protección efectiva nunca ha sido muy alta en México; e inclusive los controles de cambio y de importaciones, dada la larga frontera

2 Estructura agraria y desarrollo agricola de México, Centro de Investigaciones Agrarias, México, 1970.

3 Clark Reynolds, The Mexican Economy: Twentieth-Century Structure and Growth, Nueva Haven, Connecticut, Yale University Press, 1970, p. 124. 


\section{Gráfica 1}

MÉXICo: Relación CAPITAL/Trabajo dE LA INDUSTRIA



Fuentes: Las cifras sobre el capital provienen del Banco de México, Cuentas nacionales y acervos de capital, consolidados y por tipo de actividad económica, 1950-1967, y de A. Ortiz Mena y otros, El desarrollo económico de México y su capacidad para absorber capital del exterior, México, Nacional Financiera, 1953. Los datos sobre fuerza de trabajo provienen de DEMOgRafía y ECoNomía, vol. IV, núm. 10 y de los censos de población. 
con los Estados Unidos, nunca han sido severos en términos de las normas internacionales de países de escaso desarrollo. Pero en términos de la propia historia reciente de México, el cambio en la política que ocurrió en los años cincuenta ha servido para aislar a los empresarios industriales cada vez más de la competencia de las importaciones así como, incidentalmente, de la competencia entre ellos mismos. Como consecuencia la estructura industrial no solamente se ha vuelto más intensiva de capital, es decir, ineficiente en términos de la reserva de mano de obra desempleada (creciente), sino también se ha orientado cada vez más al mercado interno y se ha alejado del comercio internacional.

El cuadro 2 y la gráfica 2 indican que la participación de las exportaciones en el producto interno bruto ha disminuido en forma sostenida y aguda desde los años cincuenta. En términos per capita ha sucedido lo mismo (véase la gráfica 3); las exportaciones industriales bajaron en los años cincuenta a medida que la sustitución de imporración primaria, lograda mediante controles indirectos, dio paso a la sustitución de importaciones secundaria, lograda por controles directos. Las exportaciones agrícolas per capita, que habían recibido el beneficio die reformas agrícolas previas y de inversiones en infraestructura realizadas en los años cuarenta, pudieron aún obtener algún crecimiento durante los años cincuenta, pero luego descendieron agudamente en los sesenta. Vale hacer notar que las exportaciones industriales abarcaron una proporción menor de las exportaciones totales en 1970 que en 1930. En otras palabras, el apoyo en la agricultura tradicional y la busca de nuevas exportaciones agrícolas pudieron durante algún tiempo, sostener la nueva industrialización orientada al mercado interno, pero el olvido del grueso del sector agrícola, en especial en el margen intensivo, se dejó sentir finalmente.

$\mathrm{Al}$ agotarse el combustible para financiar una industrialización cada vez más costosa, inclusive con el apoyo creciente del turismo, no se pudo impedir la generación de déficit comerciales cada vez mayores cubiertos por entradas de capital. En 1946. las exportaciones de bienes constituían todavía el $75 \%$ de las exportaciones totales y se redujeron a $58 \%$ en 1964. Mientras tanto, las percepciones por turismo habían aumentado del 23 al 38\% del total. Lo que resulta más significativo es el hecho de que combustibles y minerales, que en 1940 constituían todavía el $73 \%$ de las exportaciones de bienes, se habían reducido a $25 \%$ en 1960. Esto es una indicación de que mientras el sector agrícola fue, durante un tiempo, capaz de diversificar y apoyar las políticas de sustitución de importaciones a través de un esfuerzo adicional por encontrar nuevas exportaciones primarias, esto se tornó cada vez más difícil, en especial después de 1950, cuando comenzó una disminución en las exportaciones agrícolas per capita. Por consiguiente, la carga del crecimiento continuado fue trasladada cada vez más a las cuentas invisibles y a Wall Street.

Estas tendencias negativas se aceleraron a medida que México se alejó de una trayectoria de crecimiento "ideal" dominada por un crecimiento interno equilibrado, por una parte, y de las exportaciones intensivas de trabajo, basadas en la importación de las industrias de sustitución de importaciones primarias, por otra. Por ejemplo, las importaciones de bienes para alimentar el esfuerzo de sustitución secun- 
Cuadro 2

MÉxico: Cocientes de eXportación

(Millones de pesos de 1960)

\begin{tabular}{|c|c|c|c|c|c|}
\hline & 1930 & 1940 & 1950 & 1960 & 1970 \\
\hline Exportaolones totales, E & 3451 & 5548 & 8499 & 7896 & 10383 \\
\hline Produc to interno bruto, PIB & 39667 & 41934 & 81728 & 150511 & 298700 \\
\hline $\begin{array}{l}\text { Cociente de exportaciones } \\
\text { totales, } 8 / \mathrm{PIB}\end{array}$ & 10.83 & 13.23 & 10.40 & 5.25 & 3.48 \\
\hline $\begin{array}{l}\text { Cociente de exportaciones } \\
\text { agrfoolas, } \mathrm{E}_{a} / \text { PIB }\end{array}$ & 5.97 & 5.20 & 5.62 & 3.19 & 1.96 \\
\hline $\begin{array}{l}\text { Cooiente de exportaciones } \\
\text { industriales, } \mathbb{F}_{1} / \text { PIB }\end{array}$ & 4.86 & 8.03 & 4.78 & 2.06 & 1.52 \\
\hline
\end{tabular}

Fuentes: Comisión Económica para América Latina, Doc. E/CN/12, Anuarios de Comercio Exterior de México.

Gráfica 2

MÉxico: Cocientes de eXPortacióN (Millones de pesos, $1960=100$ )



Fuentes: Comisión Económica para América Latina, Doc. E/CN/12, Anuarios de Comercio Exterior de México. 
Gráfica 3

MÉxico: EXPortaciones "PER CAPITA" (Millones de pesos, $1960=100)$

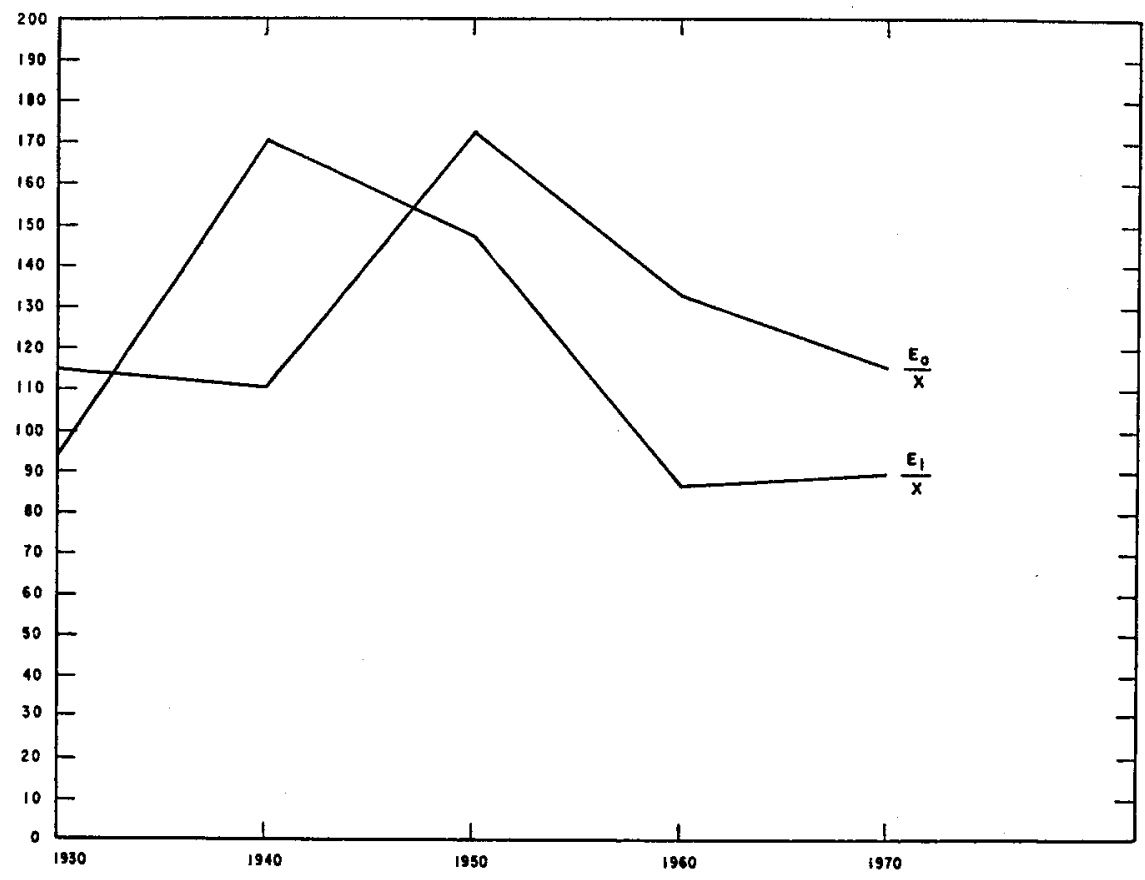

Fuentes: Comisión Económica para América Latina, Doc. E/CN/12, Anuarios de Comercio Exterior de México y censos de población.

daria aumentaron en $\$ 935$ millones entre 1960 y 1969 , mientras que las exportaciones de bienes crecieron a solamente $\$ 307$ millones durante el mismo periodo. Las percepciones invisibles, principalmente el turismo, que sirvieron para ocultar durante algún tiempo la situación, no pudieron detener la marca a mediados de los años sesenta. Al elevarse los déficit de mercancías y al crecer el turismo de mexicanos en el exterior, a una velocidad considerablemente mayor que el turismo de Estados Unidos a México, la brecha entre el déficit de mercancías y las percepciones netas por turismo alcanzó un nivel de $\$ 200$ millones anualmente entre, 1967 y 1969 y se acercó a 400 en los últimos dos años.

A medida que las exportaciones agrícolas no pudieron soportar más la carga, y el turismo tampoco pudo absorber el faltante, tuvo que acudirse cada vez más al ahorro del exterior. Según indica el cuadro 3, en 1950 México era todavía un exportador neto de capital, y desde entonces se volvió un importador neto de capital - hasta el punto en que alrededor del $14 \%$ de su inversión se financia ahora con ahorros extranjeros. Con el crecimiento elevado y sostenido entre los años cuarenta y los cincuenta, y al proseguir el sector industrial sobre la misma tendencia, México se convirtió en una especie de "niño de oro" en el desarrollo económico latinoamericano, tempranamente se graduó como concesio- 
nario de la ayuda exterior y se convirtió en objeto del mercado de bonos y de flujos de capital a corto plazo. No existe, desde luego, problema inherente en que un país en desarrollo genere un excedente de importaciones - de hecho, esto es de esperar- mientras el capital externo siga teniendo confianza en la solidez y capacidad de pago subyacentes de la economía mexicana. Lo que importa es el cambio de la estructura de la economía a medida que se desplaza hacia la fase secundaria de sustitución de importaciones y encuentra cada vez más difícil soportar el esfuerzo con ahorros generados directamente o por las exportaciones. Más aún, cada vez más el endeudamiento externo y el plazo más corto de este endeudamiento representan señales de peligro - en especial cuando vienen acompañados de una actividad de exportación menos que boyante. Los pagos anuales de deuda representan ahora alrededor de $d l s .500$ millones, $15 \%$ de las percepciones anuales por exportaciones. Al examinar más ampliamente la economía mexicana, hemos llegado así a reconocer que lo que se tiene no es esencialmente un caso de crecimiento donde algo salió mal; más bien, la senda que ha seguido la economía, durante cierto tiempo, al menos desde principios de los años cincuenta, necesita ser reexaminada y reestablecida.

\section{Cuadro 3}

México: Financiamiento externo (Millones de dólares a precios corrientes)

\begin{tabular}{|c|c|c|c|c|}
\hline & 1940 & 1950 & 1960 & 1970 \\
\hline Ahorro del exterior, $S_{f}$ & $-\quad 27$ & -33 & +340 & +1035 \\
\hline Inversión total, I & 147 & 635 & 2358 & 7500 \\
\hline$S_{f} / I$ & -18.37 & -5.20 & +14.42 & +13.80 \\
\hline
\end{tabular}

Fuentes: Comisión Económica para América Latina y Nacional Financiera, Industrial Policy in Mexican Economic Development, 1971; Clark W. Reynolds, The Mexican Economy: Twentieth-Century Structure and Growth, New Haven, Conn., Yale University Press, 1970; y Fondo Monetario Internacional, "Estadísticas Financieras Internacionales".

Hoy en día, la distribución personal del ingreso en México es más sesgada que la de casi cualquier otro país del mundo, con excepción posible del Brasil. Lo que es todavía más significativo es el hecho de que la distribución del ingreso se ha vuelto más desigual entre 1950 y 1963. En 1950 el $20 \%$ de la población de ingresos mayores recibía alrededor de 10 veces más ingreso que el 20\% inferior. En 1950, 60\% de la población recibía el $24.6 \%$ del ingreso personal; en 1963, solamente el $21.5 \%$. De este modo, México no solamente queda colocado más abajo del resto de los países en desarrollo, sino también la situación ha empeorado cada vez más. No existen datos comparables para 1970 , pero ciertamente la impresión es que esta tendencia adversa ha continuado. Sabemos, por ejemplo, que en 1969 el 10\% de la población recibía el $52 \%$ del ingreso total en comparación con el $49 \%$ en 1950 , mientras que el $40 \%$ de más bajos ingresos recibía el $11 \%$, en comparación con el $14.3 \%$ al principio. 
Con respecto al desempleo y el subempleo, toda evidencia disponible señala que el desempleo es elevado y creciente - principalmente la forma de desempleo rural en los ejidos y las pequeñas propiedades. En los años por venir puede esperarse un crecimiento demográfico y uno todavía mayor de la fuerza de trabajo, dada la estructura de edades de la población. Mientras tanto, se espera que la tasa anual de absorción de fuerza de trabajo por parte del sector industrial, que excedió a $4 \%$ en los decenios anteriores, descienda a menos de $3 \%$ a medida que continúen las tendencias actuales en la estructura de la producción y la tecnología, dictadas por la trayectoria de sustitución secundaria de importaciones en marcha. Se espera, por ejemplo, que la fuerza de trabajo aumente en 6 millones entre 1970 y 1980 y que las industrias solas no puedan absorber a más de una tercera parte de la cifra anterior.

En resumen, el camino elegido en el pasado y en el ambiente político presente, que es la consecuencia natural de la decisión original, han colocado a México en una especie de camisa de fuerza. El país se torna cada vez menos competitivo en los mercados internacionales de productos industriales - aun cuando siempre que decidió hacerlo haya podido "darse a conocer" vía el subsidio a líneas de exportación específicas (por ejemplo automóviles ensamblados en México). Los costos de ignorar el creciente endeudamiento deben contabilizarse no solamente en términos de la reducción de la eficiencia y la producción sacrificada, sino también en términos del costo humano a medida que continúa elevándose el desempleo y la distribución del ingreso se deteriora cada vez más. Solamente a través de sustituir exportaciones tradicionales por industriales podrá México escapar del desariollo del pasado, basado en la tierra, hacia un desarrollo alimentado por el uso de su abundante fuerza de trabajo no calificada, así como, un poco más tarde, incorporar la calificación, el ingenio y el capital internos al servicio de su mercado interno y externo. Las políticas que aparecen actualmente en los libros garantizan no solamente la perpetuación de un aumento en la brecha entre el crecimiento del producto y del empleo y los objetivos de distribución del ingreso, sino que, de hecho, amenazan el proceso mismo de crecimiento. Lo que se requiere es un ablandamiento sustancial del régimen de controles de cambio exterior presente a través de la liberalización de las importaciones y el ajuste al tipo de cambio, acoplado a medidas para dar a las industrias pequeña y mediana acceso más competitivo al crédito $\mathrm{y}$ un mejoramiento de los términos del intercambio del sector agrícola. Tales medidas son políticamente difíciles de poner en marcha ya que amenazan las ganancias fáciles de la élite industrial actual (interna y externa) y el poder de la burocracia. Pero los intentos por atacar el desempleo y la distribución del ingreso "directamente", sin atacar la camisa de fuerza estructural básica en que México se encuentra, representan un caso de aplicación de compresas a una fiebre muy elevada. Mientras que la reestructuración propuesta pueda no ser suficiente, y se requieran medidas directas adicionales, lo primero es ciertamente necesario para colocar a la economía en una nueva senda hacia un mayor empleo e igualdad sin sacrificar (y de hecho acelerando) el crecimiento. La ausencia de tal cambio es solamente cuestión de tiempo cuando explote la situación - ya sea porque Wall Street no responda al teléfono o porque inclusive esta tela revolucionaria ingeniosamente cosida pueda llegar a romperse. 